\title{
Two-qubit quantum probes for the temperature of an Ohmic environment
}

\author{
Francesca Gebbia $\odot,{ }^{1,2}$ Claudia Benedetti $\odot,{ }^{3}$ Fabio Benatti,,${ }^{1,2}$ Roberto Floreanini, ${ }^{2}$ Matteo Bina, ${ }^{3}$ and Matteo G. A. Paris ${ }^{3}$ \\ ${ }^{1}$ Department of Physics, University of Trieste, I-34151 Trieste, Italy \\ ${ }^{2}$ Istituto Nazionale di Fisica Nucleare, Sezione di Trieste, I-34151 Trieste, Italy \\ ${ }^{3}$ Quantum Technology Lab, Dipartimento di Fisica Aldo Pontremoli, Università degli Studi di Milano, I-20133 Milano, Italia
}

(Received 10 December 2019; accepted 28 February 2020; published 17 March 2020)

\begin{abstract}
We address a particular instance where open quantum systems may be used as quantum probes for an emergent property of a complex system, as the temperature of a thermal bath. The inherent fragility of the quantum probes against decoherence is the key feature making the overall scheme very sensitive. The specific setting examined here is that of quantum thermometry, which aims to exploit decoherence as a resource to estimate the temperature of a sample. We focus on temperature estimation for a bosonic bath at equilibrium in the Ohmic regime (ranging from sub-Ohmic to super-Ohmic), by using pairs of qubits in different initial states and interacting with different environments, consisting either of a single thermal bath or of two independent ones at the same temperature. Our scheme involves pure dephasing of the probes, thus avoiding energy exchange with the sample and the consequent perturbation of temperature itself. We discuss the role of correlations among the probes and the presence of a local versus a global bath. We show that entanglement improves thermometry at short times if the two qubits are embedded in a common bath, whereas if the interaction time is not constrained, then coherence rather than entanglement is the key resource in quantum thermometry.
\end{abstract}

DOI: 10.1103/PhysRevA.101.032112

\section{INTRODUCTION}

Quantum sensing techniques are among the most advanced quantum technologies and have led to major changes in the field of metrology in the past two decades. Upon exploiting the peculiar features of quantum systems, several novel enhanced sensors and measuring devices have been indeed suggested and demonstrated. In particular, quantum sensing based on quantum coherence and entanglement have been exploited to overcome precision bounds of classical sensors [1-4]. Among quantum sensing techniques, the use of quantum probes has recently gained attention as a noninvasive technique to estimate parameters of interest without perturbing too much the system under investigation. The basic scheme is the following: A simple quantum system, say a qubit or a pair of qubits, is prepared in a given initial state and then interacts with an external system under investigation. After the interaction, which imprints information of some parameter onto the state of the quantum probe, the latter is measured to extract such information [5-9].

In this paper, we address the use of quantum probes to estimate an emergent property of a complex system, i.e., its temperature [10-19]. In particular, in view of its importance for several fields of quantum information science, we consider here quantum thermometry of a bosonic bath in the Ohmic regime, ranging from sub-Ohmic to super-Ohmic, which induces a dephasing dynamics on the probe system [20-23]. The importance of studying purely dephasing maps resides in the fact that this model allows us to focus on the effects of quantum decoherence on thermometry, neglecting the energy exchanges between the probe and the bath [24-27]. Dephasing induces a purely quantum dynamics of the probe with no classical analog. At first sight, quantum features may not be expected to play a role in building an effective thermometer. After all, temperature is an inherently classical parameter and any change in the temperature of a sample is just changing its (classical) equilibrium distribution. However, quantum probing involves out-of-equilibrium states and since temperature is governing the amount of thermal fluctuations, the inherent fragility of quantum systems against decoherence is the key feature making the overall thermometric scheme very sensitive.

The use of single qubit as a thermometer has been analyzed recently, illustrating the interplay between the dephasing dynamics and the Ohmic structure of the environment in determining the overall precision [28,29]. Our aim is to generalize this model to study the effect of quantum correlations on thermometry. Here, we devote attention to the role of correlations in the estimation procedure and consider both the use of correlated probes as well as thermometry of correlated environments. In particular, we investigate the use of twoqubit quantum probes to estimate the temperature of different classes of bosonic systems at equilibrium, either made of a single thermal bath interacting with the two qubits or of two independent environments having the same temperature, each one interacting locally with one of the qubits. We compare performances with those obtained with a single-qubit probe and analyze in some details the interplay among the structure of the baths, the interaction time, and the quantum correlations between the qubits in determining the overall thermometric precision.

The paper is structured as follows. In Sec. II we briefly review the main concepts of quantum estimation theory that will be used throughout the work. In Sec. III we present the physical model of a two-qubit system interacting with a bosonic thermal bath, and we analyze how its dynamics is affected by a 
common environment or two independent and identical baths. In Sec. IV we assess the role of entanglement in the precision of the probing strategy, and then in Sec. V we investigate the form of the POVM that guarantees optimal performances in possible experimental implementations. Moreover, we test the robustness of the probes against initial perturbations, and we compare the performances of Bell/product states with more general probes. Section VI closes the paper with some final remarks.

\section{TOOLS OF QUANTUM ESTIMATION THEORY}

In several sensing schemes, there is no direct access to the physical quantities of interest, which need to be evaluated by means of indirect measurements. In turn, inferring the value of the quantity of interest by inspecting a set of data coming from the measurement of a different observable is precisely the goal of estimation theory.

Here we discuss how to optimally estimate the temperature $T$ of a (bosonic) thermal bath by performing a measurement on a quantum probe which is let interact with the bath, considered as its environment. The probe state is described by a density operator $\rho$ which, thanks to the interaction with the environment, becomes a function of the temperature of the bath $\rho \rightarrow \rho_{T}$. In other words, $T$, the thermodynamical temperature of the bath, becomes just a parameter of the probe state having nothing to do with a possible probe temperature. This situation is at variance with classical thermometry, where the probe is let interact with the sample until it reaches equilibrium. Then the temperature read from the probe represents the thermodynamical temperature of both the sample and the probe. The larger set of available states and the inherent fragility of quantum states against decoherence make quantum thermometry potentially more precise than any classical protocol.

To determine the parameter $T, M$ repeated measurements of a probe observable $X$ are performed on identical preparations of the probe and the outcomes $\bar{x}=\left\{x_{1}, x_{2}, \ldots, x_{M}\right\}$ are then used to construct an estimator $\widehat{T}(\bar{x})$ of the temperature $T$. Hence, data will be distributed around the mean value of the estimator according to the probability distribution $p(\bar{x} \mid T)=\prod_{k=0}^{M} p\left(x_{k} \mid T\right)$ (which is the conditional probability of obtaining the outcome $x_{k}$ when the parameter has value $T$ ) [2]:

$$
\langle T\rangle=\int d \bar{x} p(\bar{x} \mid T) \widehat{T}(\bar{x}),
$$

with a certain variance $\sigma_{T}^{2}$, which characterizes the precision of the parameter estimation. At a classical level, for unbiased estimators, the variance of such distribution is bounded from below according by the Cramèr-Rao inequality [30]:

$$
\sigma_{T}^{2} \geqslant \frac{1}{M F(T)},
$$

where $F(T)$ is the Fisher information for a single outcome,

$$
F(T)=\int d x \frac{1}{p(x \mid T)}\left(\frac{\partial p(x \mid T)}{\partial T}\right)^{2} .
$$

In turn, $F(T)$ quantifies the amount of information carried by the random variable $x$ about the unknown parameter $T$. Upon optimizing over all the possible quantum measurements one obtains the quantum Cramèr-Rao bound

$$
\sigma_{T}^{2} \geqslant \frac{1}{M F(T)} \geqslant \frac{1}{M H(T)},
$$

where $H(T)$ is the quantum Fisher information (QFI from now on). One of its explicit forms is

$$
H(T)=2 \sum_{m, n} \frac{\left|\left\langle\psi_{m}\left|\partial_{T} \rho_{T}\right| \psi_{n}\right\rangle\right|^{2}}{\lambda_{m}+\lambda_{n}},
$$

with $\psi_{n}$ and $\lambda_{n}$ being, respectively, the $T$-dependent eigenvectors and eigenvalues of $\rho_{T}$. Clearly, the optimal measurement is the one maximizing the QFI, while the the optimal estimator is that saturating the inequality.

The QFI can be written in the following form as well:

$$
H(T)=\operatorname{tr}\left(\rho_{T} L_{T}^{2}\right)
$$

where the symmetric logarithmic derivative (SLD) is given by

$$
L_{T}=2 \sum_{m, n} \frac{\left\langle\psi_{m}\left|\partial_{T} \rho_{T}\right| \psi_{n}\right\rangle}{\lambda_{m}+\lambda_{n}}\left|\psi_{m}\right\rangle\left\langle\psi_{n}\right|
$$

This object is particularly useful since it can be proven that the optimal POVM is the spectral measure of the SLD. Related to the QFI, there is another figure of merit that gives a quantification of the precision of the estimation. This is the quantum signal-to-noise ratio (QSNR), expressed as

$$
R(T)=T^{2} H(T) .
$$

The expression of the QSNR in Eq. (8) is derived from the ratio between the parameter $T^{2}$ and the (single-measure) variance of the estimator. In this way, a large value for $R$ means that the estimator has a small relative error, i.e., the error is small compared to the value of the parameter to be estimated. The QFI, QSNR, and SLD are indeed the main tools that will be used in the following.

\section{PHYSICAL MODEL}

The single-qubit case has already been described in previous works, dealing with the purely dephasing bath, related both to the estimation of temperature or other bath parameters $[28,29,31,32]$. Here we focus, under the same conditions, on the two-qubit probe scenario, which allows us to explore the role of quantum correlations and the number of qubits in inferring the temperature. We assume that initially the global state is separable $\rho(0)=\rho_{S}(0) \otimes \rho_{B}(0)$, where $\rho_{B}(0)$ is a thermal state of the environment at inverse temperature $\beta$ and characterized by the spectral density:

$$
J_{s}(\omega, \Omega)=\frac{\omega^{s}}{\Omega^{s-1}} e^{-\omega / \Omega},
$$

where $\Omega$ is the cutoff frequency and $s$ is a Ohmicity parameter which distinguishes between sub-Ohmic $(s<1)$, Ohmic $(s=1)$, and super-Ohmic $(s>1)$ regime. Spectral densities describe which bath's frequencies are coupled to the system. For $s=1$, the coupling to low frequencies increases linearly (for $\omega<\Omega$ ). Sub- and super-Ohmic spectral densities can thus be seen as a low- and band-pass filters, respectively. Depending on the physical system under investigation, the 
Ohmicity parameter $s$ may assumes different values. For instance, free background gas in one, two, or three dimensions is described by sub-Ohmics, Ohmic, and super-Ohmic, respectively [33,34]. Other examples that describe the interaction with phonon baths (Ohmic) or electromagnetic fields (subOhmic) can be found in Refs. [35-37]. Since we want to make our analysis as general as possible, we consider $s$ as a free parameter and we analyze its role in the determining the maximal extractable information from the environment.

The probe interacting with the environment is composed of two qubits, which can be in a product or entangled initial state. Moreover, the two qubits can be embedded in a common environment or in independent local baths.

\section{A. Common bath}

The Hamiltonian of two qubits interacting with a same bosonic environment can be written as

$\mathcal{H}=\sum_{j=1}^{2} \frac{\omega_{0}^{(j)}}{2} \sigma_{3}^{(j)}+\sum_{k=0}^{\infty} \omega_{k} b_{k}^{\dagger} b_{k}+\sum_{j=1}^{2} \sum_{k=0}^{\infty} \sigma_{3}^{(j)}\left(g_{k} b_{k}^{\dagger}+g_{k}^{*} b_{k}\right)$

where the index $j$ labels the qubits and the index $k$ labels the modes of the bath. $\sigma_{3}^{(j)}$ is the third Pauli matrix and $b\left(b^{\dagger}\right)$ are the annihilation (creation) bosonic operators. The $\omega_{0}^{(j)}$ are the two qubits proper frequencies, here assumed to be the same, and $\left\{\omega_{k}\right\}$ are the bath frequencies. The dephasing dynamics generated from the spin-boson model in Eq. (10) is analytically solvable $[20,21]$ and no further approximations on the dynamics are required. Moving to the continuum $\sum_{k} g_{k} \rightarrow$ $\int d \omega J_{s}(\omega, \Omega)(2|g(\omega)|)^{-2}$ and letting the compound system probe plus bath evolve up to rescaled dimensionless time $\tau=\Omega t$ according to the above Hamiltonian, after tracing over the bath's degrees of freedom, one finds that the dynamics of the two-qubit reduced density matrix can be expressed as [38]

$$
\rho_{\mathrm{CB}}(\tau, T)=\mathcal{V}(\tau, T) \circ \mathcal{R}(\tau) \circ \rho,
$$

where CB stands for "common bath" and $\mathcal{V}(\tau, T)$ is given by

$$
\mathcal{V}(\tau, T)=\left(\begin{array}{cccc}
1 & e^{-\Gamma_{s}(\tau, T)} & e^{-\Gamma_{s}(\tau, T)} & e^{-4 \Gamma_{s}(\tau, T)} \\
e^{-\Gamma_{s}(\tau, T)} & 1 & 1 & e^{-\Gamma_{s}(\tau, T)} \\
e^{-\Gamma_{s}(\tau, T)} & 1 & 1 & e^{-\Gamma_{s}(\tau, T)} \\
e^{-4 \Gamma_{s}(\tau, T)} & e^{-\Gamma_{s}(\tau, T)} & e^{-\Gamma_{s}(\tau, T)} & 1
\end{array}\right)
$$

and

$$
\mathcal{R}(\tau)=\left(\begin{array}{cccc}
1 & e^{2 i f(\tau)} & e^{2 i f(\tau)} & 1 \\
e^{-2 i f(\tau)} & 1 & 1 & e^{-2 i f(\tau)} \\
e^{-2 i f(\tau)} & 1 & 1 & e^{-2 i f(\tau)} \\
1 & e^{2 i f(\tau)} & e^{2 i f(\tau)} & 1
\end{array}\right)
$$

and $\circ$ is the Hadamard (entrywise product), while $\rho$ is the initial state of the qubits.

The decoherence function depends on time and the dimensionless temperature $T=(\Omega \beta)^{-1}$. By expressing also $\omega$ in unit of $\Omega$, i.e. $\omega \rightarrow \omega / \Omega$, we may write

$$
\Gamma_{s}(\tau, T)=\int_{0}^{\infty} d \omega e^{-\omega} \frac{1-\cos \omega \tau}{\omega^{2-s}} \operatorname{coth}\left(\frac{\omega}{2 T}\right),
$$

whose analytic expression can be found in [29]. The function $f(\tau)$ is instead a temperature-independent quantity

$$
f(\tau)=\frac{1}{2} \int_{0}^{\infty} d \omega J_{s}(\omega) \frac{\omega \tau-\sin \omega \tau}{\omega^{2}} .
$$

The matrix $\mathcal{V}(\tau, T)$ is dominant at short times and the matrix $\mathcal{R}(\tau)$ is able to create entanglement between the qubits.

\section{B. Local baths}

In the case the two qubits interact with identical but independent local environments, the Hamiltonian takes the form

$$
\mathcal{H}=\sum_{j=1}^{2}\left[\frac{\omega_{0}^{(j)}}{2} \sigma_{3}^{(j)}+\sum_{k=0}^{\infty} \omega_{k} b_{k}^{(j) \dagger} b_{k}^{(j)}+\sigma_{3}^{(j)} \sum_{k=0}^{\infty}\left(g_{k} b_{k}^{(j) \dagger}+g_{k}^{*} b_{k}^{(j)}\right)\right] \text {. }
$$

Let the initial density matrix of the bath be factorized $\rho_{b}(0)=$ $\rho_{B}^{(1)} \otimes \rho_{B}^{(2)}$, where $\rho_{B}^{(1,2)}$ are thermal Gibbs states at dimensionless temperature $T$. In this case, the evolution of the probe is given by

$$
\rho_{\mathrm{LB}}(\tau, T)=\mathcal{W}(\tau, T) \circ \rho,
$$

where LB stands for "local baths" and $\mathcal{W}(\tau, T)$ is given by

$\mathcal{W}(\tau, T)=\left(\begin{array}{cccc}1 & e^{-\Gamma_{s}(\tau, T)} & e^{-\Gamma_{s}(\tau, T)} & e^{-2 \Gamma_{s}(\tau, T)} \\ e^{-\Gamma_{s}(\tau, T)} & 1 & e^{-2 \Gamma_{s}(\tau, T)} & e^{-\Gamma_{s}(\tau, T)} \\ e^{-\Gamma_{s}(\tau, T)} & e^{-2 \Gamma_{s}(\tau, T)} & 1 & e^{-\Gamma_{s}(\tau, T)} \\ e^{-2 \Gamma_{s}(\tau, T)} & e^{-\Gamma_{s}(\tau, T)} & e^{-\Gamma_{s}(\tau, T)} & 1\end{array}\right)$.

Since the evolution of the qubits in local and identical baths is the same as sending twice a single qubit through the channel, $\mathcal{W}(\tau, T)$ is the tensor product of two single qubit dephasing maps $\mathcal{W}(\tau, T)=V(\tau, T) \otimes V(\tau, T)$, where $V(\tau, T)$ takes the form [28]

$$
V(\tau, T)=\left(\begin{array}{cc}
1 & e^{-\Gamma_{s}(\tau, T)} \\
e^{-\Gamma_{s}(\tau, T)} & 1
\end{array}\right)
$$

\section{TWO-QUBIT THERMOMETRY}

We now compare the performances of two-qubit quantum probes for the temperature by analyzing the behavior of the QFI. We consider two different initial states for the qubits, i.e., initially entangled and initially separable probes, and we study the effect of the probe interaction with a common bath against local baths. Due to the convexity properties of the QFI [39], we focus on initial pure states for the probe. More specifically, to assess the effect of entanglement, we compare the precision obtained using Bell states probe to that achievable by product states.

\section{A. Entangled qubits}

We start by analyzing the performances of quantum probes initially prepared in one of the Bell state: $\left|\varphi^{ \pm}\right\rangle=\frac{1}{\sqrt{2}}(|00\rangle \pm$ $|11\rangle)$ and $\left|\psi^{ \pm}\right\rangle=\frac{1}{\sqrt{2}}(|01\rangle \pm|10\rangle)$. We first consider the scenario with a common environment: The Bell states $\left|\psi^{ \pm}\right\rangle$are not changed by the map [Eq. (11)], while $\left|\varphi^{ \pm}\right\rangle$are affected by 
the environment. Indeed, for both states $\left|\varphi^{ \pm}\right\rangle$, the QFI is

$$
H_{\mathrm{CB}}^{\mathrm{ent}}(\tau, T, s)=\frac{\left[4 \partial_{T} \Gamma_{s}(\tau, T)\right]^{2}}{e^{8 \Gamma_{s}(\tau, T)}-1} .
$$

Notice that this quantity does not depend on $f(\tau)$. However, when the interaction of the probe with two identical and independent local baths is considered, all four Bell states lead to the same QFI:

$$
H_{\mathrm{LB}}^{\mathrm{ent}}(\tau, T, s)=\frac{\left[2 \partial_{T} \Gamma_{s}(\tau, T)\right]^{2}}{e^{4 \Gamma_{s}(\tau, T)}-1} .
$$

A numerical evaluation of the QFI shows that the Bell states $\left|\varphi^{ \pm}\right\rangle$in a global-bath scenario and any of the Bell states in local baths are indeed optimal within the subset of states

$$
\begin{gathered}
\left|\Phi_{\alpha}\right\rangle=\cos \alpha|00\rangle+\sin \alpha|11\rangle, \\
\left|\Psi_{\alpha}\right\rangle=\cos \alpha|01\rangle+\sin \alpha|10\rangle,
\end{gathered}
$$

with $\alpha \in[0,2 \pi]$ a real parameter.

\section{B. Separable qubits}

Let us consider two qubits initially prepared in the product state $|++\rangle$, where $|+\rangle=\frac{1}{\sqrt{2}}(|0\rangle+|1\rangle)$, which is a particular instance of the one-parameter family of states

$$
\left|\psi_{\alpha} \psi_{\beta}\right\rangle:=\left|\psi_{\alpha}\right\rangle \otimes\left|\psi_{\beta}\right\rangle, \quad\left|\psi_{\alpha}\right\rangle=\cos \alpha|0\rangle+\sin \alpha|1\rangle .
$$

In the common-bath case, the QFI has the expression

$$
\begin{aligned}
& H_{\mathrm{CB}}^{\mathrm{sep}}(\tau, T, s) \\
& =\frac{4\left(2-e^{2 \Gamma_{s}(\tau, T)}+2 e^{4 \Gamma_{s}(\tau, T)}+e^{6 \Gamma_{s}(\tau, T)}\right)\left[\partial_{\mathrm{T}} \Gamma_{s}(\tau, T)\right]^{2}}{3 e^{8 \Gamma_{s}(\tau, T)}-2 e^{4 \Gamma_{s}(\tau, T)}-1} .
\end{aligned}
$$

Again, the quantity $f(\tau)$ does not play a role in the estimation of the temperature. In the case of local independent baths, instead, the QFI reads

$$
H_{\mathrm{LB}}^{\mathrm{sep}}(\tau, T, s)=\frac{2\left[\partial_{\mathrm{T}} \Gamma_{s}(\tau, T)\right]^{2}}{e^{2 \Gamma_{s}(\tau, T)}-1} .
$$

As expected it is twice the QFI of a single-qubit probe [29]. Both results Eqs. (24) and (25) can be obtained by initializing the probe also in one of the states $|--\rangle,|+-\rangle$, and $|-+\rangle$.

\section{Comparison}

Let us now compare the behavior of the quantum signal-tonoise ratio (QSNR) given in Eq. (8), that for sake of simplicity we shall denote by $R$ in the following, for different initial states and bath parameters. In Fig. 1, we show the behavior of $R$ for different values of the Ohmicity parameter as a function of the dimensionless time and temperature and for the four possible scenarios described in Secs. IV A and IV B. The evaluation of the QSNR can only be performed numerically, through the integration of the decoherence function Eq. (14). From Fig. 1, a universal behavior emerges which is independent on the Ohmicity parameter. Indeed, we found two optimal scenarios that give the maximum of the QSNR $R$. For short time intervals (namely, $\tau \ll 1$ ), the optimal strategy to estimate the temperature is to employ an entangled probe in a



FIG. 1. Left column: Behavior of the QSNR for three different values of $s=0.5,1,3$, as a function of dimensionless time $\tau$ and temperature $T$. The four different colors correspond to the possible scenarios analyzed in this work, i.e., common/local bath and entangled/separable initial state. Right column: Ratio $R_{\mathrm{CB}}^{\text {ent }} / R_{\mathrm{LB}}^{\mathrm{sep}}$ for the same three values of $s$.

common bath. For longer times, the estimation is more precise if a separable initial state is left to evolve in an environment consisting of local independent baths. It follows that, if time is a resource that we need to use parsimoniously, then the best option is to use an entangled probe interacting with a common environment. On the contrary, if we can wait for longer times, then the (absolute) best strategy is sending sequentially two qubits prepared in a $|+\rangle$ state through the bath, i.e., repeating twice the single-qubit scheme [29]. In Fig. 1, we also analyze the ratio $R_{\mathrm{CB}}^{\text {ent }} / R_{\mathrm{LB}}^{\text {sep }}$ to emphasize the optimal procedures in the $(\tau, T)$-space. As we see from the graphs, the Ohmicity parameter $s$ only affects the qualitative behaviors of the ratio, and the two different estimation strategies are clearly displayed. To better understand why the change from one optimal strategy to the other occurs, we examine the behavior of the quantum Fisher information $H$ for small values of $\tau$ :

$$
H(T, \tau, s) \simeq \tau^{2} \begin{cases}\frac{1}{\gamma(T, s)}\left[\partial_{T} \gamma(T, s)\right]^{2} & \text { for } H_{\mathrm{CB}}^{\mathrm{ent}}, \\ \frac{1}{2 \gamma(T, s)}\left[\partial_{T} \gamma(T, s)\right]^{2} & \text { other cases, }\end{cases}
$$

where

$$
\Gamma_{s}(T, \tau) \simeq \frac{\tau^{2}}{2} \int_{0}^{\infty} d x x^{s} e^{-x} \operatorname{coth} \frac{x}{2 T} \equiv \frac{\tau^{2}}{2} \gamma(T, s)
$$

at short times. So, $H_{\mathrm{CB}}^{\mathrm{ent}}$ outperforms by a factor 2 the other strategies in the $\tau \ll 1$ regime. 


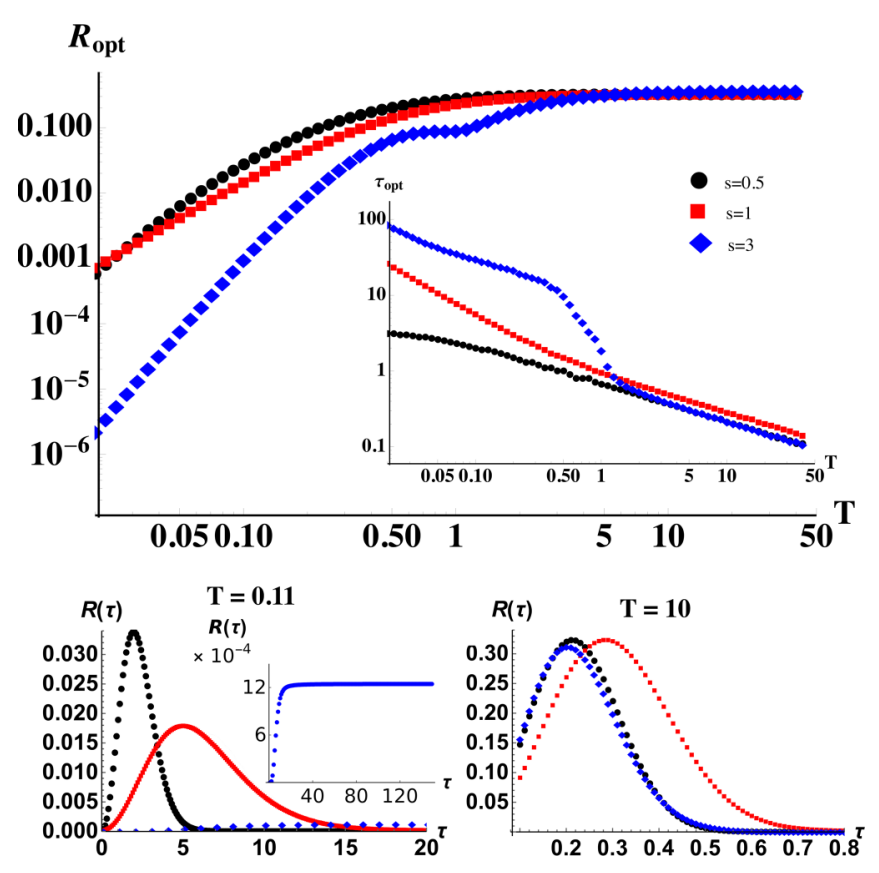

FIG. 2. Top panel: Maximum value of the QSNR in time as a function of the temperature for different values of $s$. This maximum is obtained by using separable probes in local baths, as shown in Fig. 1. In the inset, optimal time, i.e., time at which $R$ reaches the maximum value, as a function of $T$. Lower panel: time evolution of the QSNR for different values of $s$, for low (left plot) and high (right plot) temperatures. The inset in the left plot shows a zoom of the dynamics of the QSNR for the super-Ohmic $s=3$ case.

In Fig. 2, we show the maximum value of the QSNR, called $R_{\text {opt }}$, as a function of the temperature and for different values of the Ohmicitiy parameter $s$. Notice that this maximum is obtained in the case where two separable qubits are embedded in local baths. We notice that, as the Ohmicity parameter $s$ grows, the maximal values of the QSRN decrease for a fixed value of the temperature, except for $T$ becoming large and the dependency on $s$ disappears. A decreasing QFI with increasing Ohmicity parameter $s$ was also found in Ref. [40], where a quantum Brownian probe was strongly coupled with a reservoir. Large values of $s$ imply that the higher frequencies of the bath (with respect to $\omega_{c}$ ) strongly interact with the probe, while small values of the Ohmicity parameter correspond to lower frequencies coupled to the qubits. Our results thus show that if the probe interacts mainly with low frequencies of the bath, then the estimation of the temperature is very precise. On the contrary, interactions with high frequencies do not allow to extract much information about the environment. For further readings, the role of the spectral density and Ohmicity in quantum thermometry has been examined in Refs. [41,42].

The time corresponding to the maximum of $R, R_{\mathrm{opt}}$, is called optimal time $\tau_{\text {opt }}$; notice that, especially for low temperatures, it strongly depends on the Ohmicity parameter. Furthermore, Fig. 2 shows agreement with the behaviour found in the case of single qubit thermometry [29], as expected since the maximum of the QSNR is obtained employing two qubits in a product states that evolve in local baths. This procedure is the same as sending twice a single qubit into the quantum environment. For the sake of completeness, in

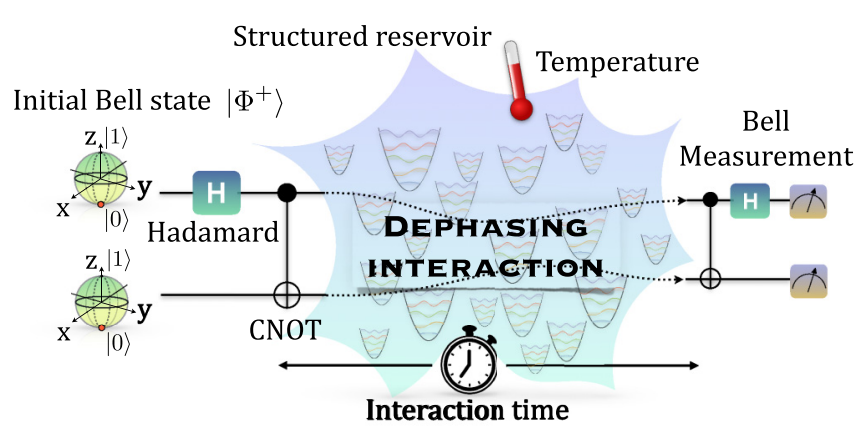

FIG. 3. General scheme for state $\left|\varphi^{+}\right\rangle$in the same bath. The optimal measurement consists in a Bell measurement along two Bell states.

the lower plots of Fig. 2, we also show typical behaviors of the QSNR as a function of time for different values of the Ohmicity parameter and different temperature regimes. At low temperatures $R(\tau)$ has a maximum in time, except for the super-Ohmic case, where a monotonic behavior is found, with the QSNR that reaches a constant value after a certain time. At higher temperatures, instad, the QSNR always has a single maximum in time, in all Ohmicity regimes.

\section{IMPLEMENTATIONS}

In this section we analyze possible ways to experimentally implement the optimal strategies. We focus on entangled qubits in a common bath at short times, and separable qubits in independent baths for longer interaction times. By calculating the symmetric logarithmic derivative, we derive the optimal POVM to be performed on the qubits to infer the value of temperature in a bosonic environment.

\section{A. SLD for Bell states in a common bath}

We begin by considering the scenario of the optimal Bell states $\left|\varphi^{ \pm}\right\rangle$in a common bath. The SLD is derived using Eq. (7):

$$
L_{\mathrm{CB}}^{\mathrm{ent}}=a_{-}\left|\varphi^{-}\right\rangle\left\langle\varphi^{-}\left|-a_{+}\right| \varphi^{+}\right\rangle\left\langle\varphi^{+}\right|,
$$

where

$$
a_{ \pm}=\frac{4 \partial_{\mathrm{T}} \Gamma_{s}(\tau, T)}{e^{4 \Gamma_{s}(\tau, T)} \pm 1} .
$$

The coefficients of the decomposition depend on the temperature, time and the Ohmicity, but the projectors are temperature- and Ohmicity-independent. The optimal protocol thus requires to experimentally discriminate the Bell states $\left|\varphi^{ \pm}\right\rangle$. This general scheme is sketched in Fig. 3 .

\section{B. SLD for $|++\rangle$ states in local baths}

Regarding the separable state $|++\rangle$ coupled with two independent local baths, the SLD has the expression

$$
L_{\mathrm{LB}}^{\text {sep }}=b_{-} \Pi_{-}-b_{+} \Pi_{+}+c\left(\left|\varphi^{-}\right\rangle\left\langle\varphi^{-}|+| \psi^{-}\right\rangle\langle\psi-|\right),
$$

where

$$
b_{ \pm}=\frac{2 \partial_{\mathrm{T}} \Gamma_{s}(T, \tau)}{e^{\Gamma_{s}(T, \tau)} \pm 1}, \quad c=\frac{2 \partial_{\mathrm{T}} \Gamma_{s}(T, \tau)}{e^{2 \Gamma_{s}(T, \tau)}-1}
$$




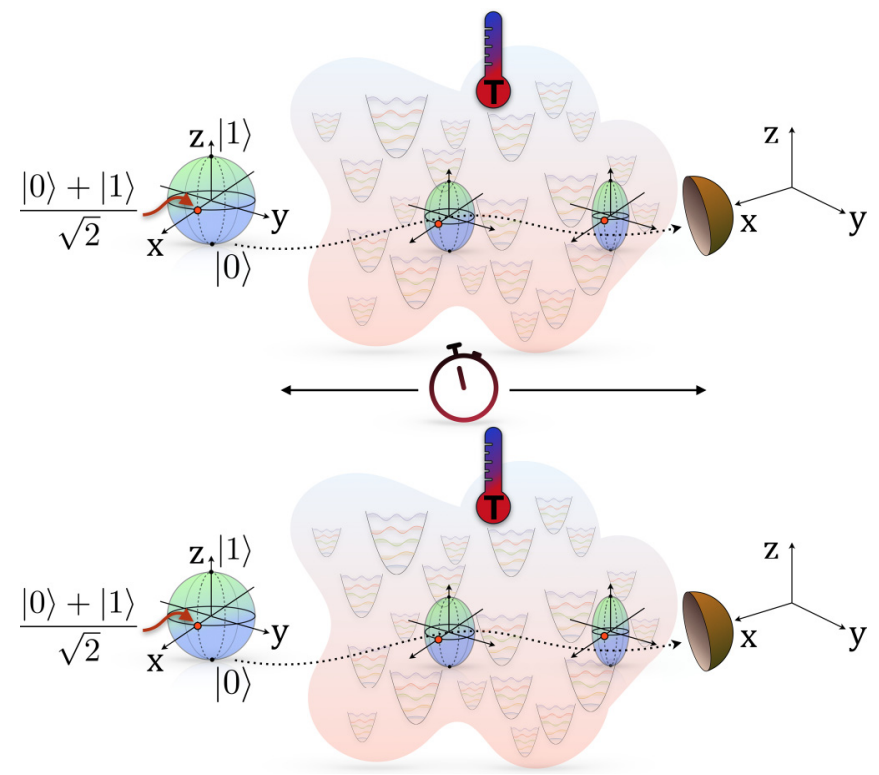

FIG. 4. General scheme of two qubits in the $|++\rangle$ state interacting with independent baths and being measured separately along $\sigma_{1}$.

and $\Pi_{ \pm}=| \pm \pm\rangle\langle \pm \pm|$. Although the presence of Bell states in Eq. (30) may led to think that global measurements on both qubits are needed to discriminate the various projections, actually this is not the case: the eigenstates of $L_{\mathrm{LB}}^{\text {sep }}$ are eigenvectors of $\sigma_{1} \otimes \sigma_{1}$, an observable that can be accessed locally, namely, by separate measurements on the two qubits. This scenario is sketched in Fig. 4.

\section{Robustness}

We want to check the robustness of the optimal states, namely, we want to quantify how the maximal QFI is affected by small deviations $\delta$ from the optimal states. In particular, we consider purity-preserving deviations, since we are primarily interested in investigating the role of inaccuracy on the state preparation. Indeed, we want to analyze how a small difference in the optimal parameters affects the precision of the estimation.

We start by focusing on the two optimal scenarios. As already mentioned, sending two qubits in a product state in independent local environments corresponds to repeating the same experiment twice; therefore, we can write the QFI for a generic initial state $\left|\psi_{\alpha} \psi_{\beta}\right\rangle$ defined in Eq. (23) as the sum of the QFI of the two single qubits:

$$
H_{\mathrm{LB}}^{\alpha \beta}(\tau, T)=\left[\sin ^{2}(2 \alpha)+\sin ^{2}(2 \beta)\right] \frac{\left[\partial_{T} \Gamma_{s}(\tau, T)\right]^{2}}{e^{2 \Gamma_{s}(\tau, T)}-1} .
$$

We now consider the perturbed initial state $\left|\psi_{\alpha+\delta_{\alpha}} \psi_{\beta+\delta_{\beta}}\right\rangle$ around the optimal choice $(\alpha=\beta=\pi / 4)$, we find the perturbed QFI:

$$
H_{\mathrm{LB}}^{\delta_{\alpha} \delta_{\beta}}(\tau, T)=H_{\mathrm{LB}}^{\alpha \beta}(\tau, T)\left[1-2\left(\delta_{\alpha}^{2}+\delta_{\beta}^{2}\right)\right] .
$$

On the other side, the general entangled state $\left|\Phi_{\alpha}\right\rangle$ defined in Eq. (21) in a common bath yields

$$
H_{\mathrm{CB}}^{\alpha}(\tau, T)=\frac{16 \sin ^{2}(2 \alpha)\left[\partial_{\mathrm{T}} \rho_{\mathrm{T}}\right]^{2}}{e^{8 \Gamma_{s}(\tau, T)}-1} .
$$

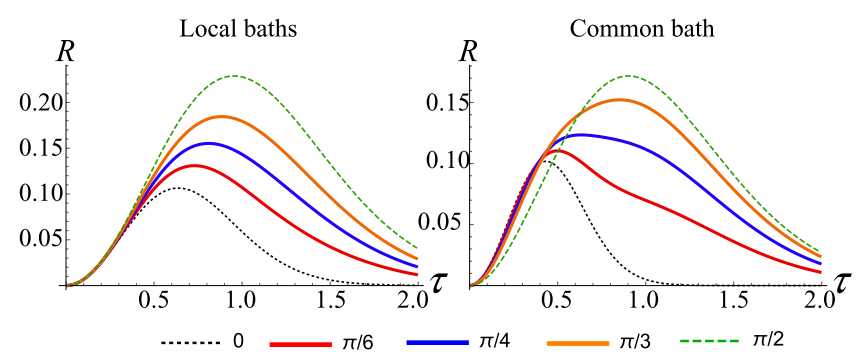

FIG. 5. QSNR $R$ as a function of time for $s=1, T=1$ and different values of the parameter $\alpha$ in Eq. (36). Both local- and common-bath scenarios are represented.

If we perturb the state $\left|\Phi_{\alpha}\right\rangle$ around $\alpha=\pi / 4$, then we find the following QFI:

$$
H_{\mathrm{CB}}^{\delta}(\tau, T) \simeq H_{\mathrm{CB}}^{\mathrm{ent}}(\tau, T)\left(1-4 \delta^{2}\right) .
$$

In both cases the deviation from the optimal QFI are at second order on the magnitude of the perturbation, i.e., the QFI obtained from both $\left|\varphi^{+}\right\rangle$and $|++\rangle$is robust with respect to the probe preparation.

\section{Performances of other probes}

So far we maximized the QFI within specific subsets of state, $\left|\Phi_{\alpha}\right\rangle$ and $\left|\psi_{\alpha} \psi_{\beta}\right\rangle$ for entangled and separable probes and we found that, within these families, the states $\left|\varphi^{+}\right\rangle$and $|++\rangle$ are optimal regardless of $\tau$ and $T$. We now consider the normalized superposition:

$$
\left|S_{\alpha}\right\rangle=C\left(\cos \alpha\left|\varphi^{+}\right\rangle+\sin \alpha|++\rangle\right),
$$

where $C$ is a normalization constant. In Fig. 5 we compare the behavior of the QSNR for $s=1$ and $T=1$ for different values of the parameter $\alpha$ in Eq. (36). Both the local and global scenarios are analyzed. The largest value of the QSNR is obtained for $\alpha=\pi / 2$, corresponding to initially separable qubits. At shorter times, the $\alpha=0$ case, i.e., entangled qubits, is the best probe, but only in the common-bath case. In the common-environment scenario, moreover, there is also a small time-interval where other probes perform better, while this behavior is never seen in the local-bath case.

To investigate the role of other probes, we compare the QSNR for randomly-generated pure states. In Fig. 6, we
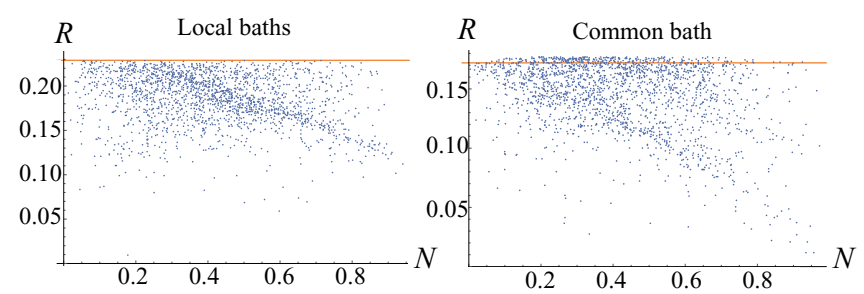

FIG. 6. Maximum value of the QSNR $R$ as a function of the entanglement $N$ of the initial state of the probes in a time interval $\tau \in[0.2] .2000$ pure states are generated randomly, both for the local- and common-bath scenario. We set $s=1$ and $T=1$. The orange line is a guide for the eye for the maximum value of the QSNR reached by the probe $|++\rangle$. 
display the behavior of the maximum of the QSNR in a time interval $\tau \in[0,2]$ as a function of the entanglement of initial state of the probe. We generated 2000 points, representing different initial pure states with a different level of entanglement. Entanglement is evaluated through the negativity $N$ [43]. We see that, in the local-bath scenario, the separable state $|++\rangle$ achieves indeed the maximum of the QSNR, marked by the orange line. All other generated states have a QSNR that stays below this value. However, this is not the case in the common-bath scenario, where there exist entangled probes that perform better than the $|++\rangle$ state and that cannot be described through Eq. (36). These states, in fact, outperform the product state in estimating the temperature of the bath, as shown on the right plot of Fig. 6.

\section{CONCLUSIONS}

The aim of thermometry is to estimate the temperature of a certain object without altering its properties, temperature included. When the object is a quantum system, the idea of finding a noninvasive probe becomes a necessity, to avoid decoherence. In this paper, we have addressed optimal quantum thermometry for bosonic thermal baths by means of two-qubit quantum probes. We have analyzed the case of two independent baths, each interacting locally with a qubit, and the case of a common reservoir acting upon the two qubits. Moreover, we have considered two families of initial states for the probes: Bell states and product states of the form $|\sigma, \eta\rangle$ where $\sigma= \pm$ and $\eta= \pm$. In particular, we have analyzed the behavior of the QFI and the QSNR as a function of time, temperature and ohimicity parameter. Our results show that at short times the best way to probe the temperature of the bath is to employ maximally entangled qubit interacting with the same global bath, independently from the Ohmicity of the environment. However, if time is not considered a resource, then we found that the best estimate is obtained upon using two qubits in a product states, each one subject to a local environment, i.e., the best strategy is to repeat twice a singlequbit probe measurement [28,29]. Further investigations of the performances of multiqubit probes are necessary to go beyond the two-qubit model and study the scaling of the QFI with the number of probes.

Notice that the notions of same bath or two independent replicas of the same bath do not require multiple physical systems to be implemented in practice. In a realistic environment, the two situations correspond, respectively, to the two qubits propagating one close to each other, to sense the same portion of the environment, or far away, such that possible spatial correlations of the bath may be neglected. We have analysed possible implementations of the optimal estimation schemes discussed above and we found that the entangled case boils down to a Bell measurement over the entangled quits, while the case of factorized probe may be implemented by a local measurement performed separately on the two qubits. We have analyzed the behavior of the estimation precision against perturbations in the preparation of the initial state and have shown the they contribute to decrease the QFI with second order corrections in the perturbations, i.e., our scheme is robust.

We have also compared the performances of the Bell and product states with other probes. Our intent was to analyze whether a combination of the Bell and separable states could improve the precision of the estimation. We showed that the absolute maximum of the QFI is indeed obtained for independent qubits in a product state. However, there exists temporal regions where a superposition of the two probes perform better, though only in the common-bath scenario. To gain more insight into the role of other probes, we have randomly generated pure states and analyzed the maximum value of their QFI versus the entanglement of the generated state. We found numerical evidence that the state $|++\rangle$ is the optimal one in the case of local environments, while for the common bath there exist initial entangled states of the probe that outperform the product state in a certain temporal region that depends upon the temperature and the Ohmicity parameter. The corresponding optimal measurement, however, do not correspond to an easy implementable one and thus it may be challenging to achieve the corresponding enhancement in a practical scenario.

Summarizing our results, we have that entanglement improve thermometry at short times whereas, if the interaction time is not constrained, coherence rather than entanglement, is the key resource to improve precision. We also emphasize that our scheme for quantum thermometry is based on pure dephasing and it does not involve energy exchanges between the probes and the bath. The corresponding measuring protocols are thus inherently non invasive, and they may be of value whenever a direct inspection threatens to destroy the sample, or to perturb the temperature itself, as it would happen in the case of very cold samples.

\section{ACKNOWLEDGMENTS}

M.G.A.P. is a member of INdAM-GNFM. C.B., M.B., and M.G.A.P. thank S. Razavian, I. Amelio, and F. Grasselli for useful discussions. This work has been partially supported by JSPS through the FY2017 program (Grant No. S17118).
[1] C. L. Degen, F. Reinhard, and P. Cappellaro, Quantum sensing, Rev. Mod. Phys. 89, 035002 (2017).

[2] M. G. A. Paris, Quantum estimation for quantum technology, Int. J. Quantum Inf. 07, 125 (2009).

[3] D. Braun, G. Adesso, F. Benatti, R. Floreanini, U. Marzolino, M. W. Mitchell, S. Pirandola, Quantum-enhanced measurements without entanglement, Rev. Mod. Phys. 90, 035006 (2018).
[4] J. F. Haase, A. Smirne, S. F. Huelga, J. Kolodynski, F. Demkowicz-Dobrzanski, Precision limits in quantum metrology with open quantum systems, Quantum Meas. Quantum Metr. 5, 13 (2018).

[5] C. Benedetti, F. Buscemi, P. Bordone, and M. G. A. Paris, Quantum probes for the spectral properties of a classical environment, Phys. Rev. A 89, 032114 (2014). 
[6] G. L. Giorgi, F. Galve, and R. Zambrini, Probing the spectral density of a dissipative qubit via quantum synchronization, Phys. Rev. A 94, 052121 (2016).

[7] J. Nokkala, F. Galve, R. Zambrini, S. Maniscalco, and J. Piilo, Complex quantum networks as structured environments: Engineering and probing, Sci. Rep. 6, 26861 (2016).

[8] F. Cosco, M. Borrelli, F. Plastina, and S. Maniscalco, Momentum-resolved and correlation spectroscopy using quantum probes, Phys. Rev. A 95, 053620 (2017).

[9] A. Usui, B. Buča and J. Mur-Petit, Quantum probe spectroscopy for cold atomic systems, New J. Phys. 20, 103006 (2018).

[10] E. Martìn-Martìnez, A. Dragan, R. B. Mann, and I. Fuentes, Berry phase quantum thermometer, New J. Phys. 15, 053036 (2013).

[11] C. Sabín, A. White, L. Hackermuller, and I. Fuentes, Impurities as a quantum thermometer for a Bose-Einstein condensate, Sci. Rep. 4, 6436 (2014)

[12] M. Brunelli, S. Olivares, and M. G. A. Paris, Qubit thermometry for micromechanical resonators, Phys. Rev. A 84, 032105 (2011).

[13] L. A. Correa, M. Mehboudi, G. Adesso, and A. Sanpera, Individual Quantum Probes for Optimal Thermometry, Phys. Rev. Lett. 114, 220405 (2015).

[14] A. De Pasquale, K. Yuasa, and V. Giovannetti, Estimating temperature via sequential measurements, Phys. Rev. A 96, 012316 (2017).

[15] S. Campbell, M. G. Genoni, and S. Deffner, Precision thermometry and the quantum speed limit, Quantum Sci. Technol. 3, 025002 (2018).

[16] M. R. Jørgensen, P. P. Potts, M. G. A. Paris, and J. B. Brask, Tight bound on finite-resolution quantum thermometry at low temperatures, arXiv:2001.04096.

[17] S. Jevtic, D. Newman, T. Rudolph, and T. M. Stace, Singlequbit thermometry, Phys. Rev. A 91, 012331 (2015)

[18] M. Mehboudi, A. Sanpera and L. A. Correa, Thermometry in the quantum regime: Recent theoretical progress, J. Phys. A: Math. Theor. 52, 303001 (2019).

[19] P. P. Potts, J. B. Brask, and N. Brunner, Fundamental limits on low-temperature quantum thermometry with finite resolution, Quantum 3, 161 (2019).

[20] G. M. Palma, K.-A. Suominen, and A. Ekert, Quantum computers and dissipation, Proc. R. Soc. Lond. A 452, 567 (1996).

[21] H. P. Breuer and F. Petruccione, The Theory of Open Quantum Systems, Sec. 4.2, (Oxford University Press, Oxford, UK, 2002).

[22] X. Fan, T. Takagahara, J. E. Cunningham, and H. Wang, Pure dephasing induced by exciton-phonon interactions in narrow GaAs quantum wells, Solid State Commun. 108, 857 (1998).

[23] H. Hwang and P. J. Rossky, An analysis of electronic dephasing in the spin-boson model, J. Chem. Phys. 120, 11380 (2004).

[24] B-H. Liu, Li Li, Y.-F. Huang, C.-F. Li, G.-C. Guo, E.-M. Laine, H.-P. Breuer, and J. Piilo, Experimental control of the transition from Markovian to non-Markovian dynamics of open quantum systems, Nat. Phys. 7, 931 (2011).
[25] S. Cialdi, M. A. C. Rossi, C. Benedetti, B. Vacchini, D. Tamascelli, S. Olivares, and M. G. A. Paris, All-optical quantum simulator of qubit noisy channels, Appl. Phys. Lett. 110, 081107 (2017).

[26] H.-B. Chen, P.-Y. Lo, C. Gneiting, J. Bae, Y.-N. Chen, and F. Nori, Quantifying the nonclassicality of pure dephasing, Nat. Commun. 10, 3794 (2019).

[27] M. Bramberger and I. De Vega, Dephasing dynamics of an impurity coupled to an anharmonic environment, Phys. Rev. A 101, 012101 (2020).

[28] S. Razavian and M. G. A. Paris, Quantum metrology out of equilibrium, Physica A 525, 825 (2019).

[29] S. Razavian, C. Benedetti, M. Bina, Y. Akbari-Kourbolagh, and M. G. A. Paris, Quantum thermometry by single-qubit dephasing, Eur. Phys. J. Plus 134, 284 (2019).

[30] H. Cramér, Mathematical methods of statistic (Princeton University Press, Princeton, NJ, 1962).

[31] C. Benedetti, F. S. Sehdaran, M. H. Zandi, and M. G. A. Paris, Quantum probes for the cut-off frequency of Ohmic environments, Phys. Rev. A 97, 012126 (2018).

[32] F. Salari Sehdaran, M. Bina, C. Benedetti, and M. G. A. Paris, Quantum probes for Ohmic environments at thermal equilibrium, Entropy 21, 486 (2019).

[33] M. A. Cirone, G. De Chiara, G. M. Palma, and A. Recati, Collective decoherence of cold atoms coupled to a Bose-Einstein condensate, New J. Phys. 11, 103055 (2009).

[34] P. Haikka, S. McEndoo, G. De Chiara, G. M. Palma, and S. Maniscalco, Quantifying, characterizing, and controlling information flow in ultracold atomic gases, Phys. Rev. A 84, 031602(R) (2011).

[35] U. Weiss, Quantum Dissipative Systems (World Scientific Publishing, Singapore, 1999).

[36] A. Shnirman, Y. Makhlin, and G. Schön, Noise and decoherence in quantum two-level systems, Phys. Scr. T102, 147 (2002).

[37] J. Paavola, J. Piilo, K.-A. Suominen, and S. Maniscalco, Environment-dependent dissipation in quantum Brownian motion, Phys. Rev. A 79, 052120 (2009).

[38] J. H. Reina, L. Quiroga, and N. F. Johnson, Decoherence of quantum registers, Phys. Rev. A 65, 032326 (2002).

[39] R. Demkowicz-Dobrzański, M. Jarzyna, and J. Kołodyński, Quantum limits in optical interferometry, Prog. Opt. 60, 345 (2015).

[40] L. A. Correa, M. Perarnau-Llobet, K. V. Hovhannisyan, S. Hernàndez-Santana, M. Mehboudi, and A. Sanpera, Enhancement of low-temperature thermometry by strong coupling, Phys. Rev. A 96, 062103 (2017).

[41] K. V. Hovhannisyan and L. A. Correa, Measuring the temperature of cold many-body quantum systems, Phys. Rev. B 98, 045101 (2018).

[42] A. A. Valido, A. Ruiz, and D. Alonso, Quantum correlations and energy currents across three dissipative oscillators, Phys. Rev. E 91, 062123 (2015).

[43] G. Vidal and R. F. Werner, Computable measure of entanglement, Phys. Rev. A 65, 032314 (2002). 